\title{
A Randomized Double-Blind Study: Evulation of Comparing Intravenous Fentanyl with Intravenous Tramadol Administered to Patients with Pain Control Due to Urinary Stone Disease
}

\author{
(D) Abdullah Osman Koçak
}

Department of Emergency Medicine, Atatürk University Faculty of Medicine, Erzurum, Turkey

\begin{abstract}
Aim: The aim of our study is to compare the efficiency of fentanyl and tramadol as analgesics in renal colic patients.

Materials and Methods: Our research is a prospective, randomized, double-blind study. We administered intravenous (i.v.) non-steroid anti-inflammatory drugs as first line treatment. Then, "i.v. fentanyl" and "i.v. tramadol" were administered to the patients whose pain did not relieve. The outcomes of the analgesic efficacy in our study were the difference between VAS 0-VAS 60 values and the score less than 4 in VAS 60

Results: A total of 912 renal colic patients were admitted during the study period. When the exclusion criteria were applied, the study was completed with 143 patients. The difference between VAS 0 and VAS 60 values-which is the primary outcome in the evaluation of analgesic efficiency in our study is $6.11 \pm 2.49$ in fentanyl and $5.94 \pm 2.40$ in tramadol group. While 16 patients responded to the treatment in the fentanyl group, 18 patients responded to the treatment in the tramadol group.
\end{abstract}

Conclusion: Our study concluded that both drugs are effective on renal colic pain and they do not have a significant advantage over each other.

Keywords: Renal colic, fentanyl, tramadol

\section{Introduction}

Pain is a global public health problem and is the most common cause of admission to the hospital. If the pain cannot be managed properly, it causes medical complications, chronic pain development and low patient satisfaction. Pain management is still a challenge for healthcare professionals today. These difficulties include the problems associated with the interaction of drugs, side effects and addiction to some drugs. Therefore, maximum care should be taken in pain management (1).

Acute flank pain is a common and complex clinical problem that most frequently caused by urolithiasis and also can be caused by urinary and extra-urinary anomalies (2). Renal colic is usually defined as acute pain in the flank due to the passing of a stone through the ureter.

Acute renal colic is a pain that starts in the flank and spreads to the groin. Renal colic is often accompanied with microscopic hematuria ( $85 \%$ of patients), nausea, vomiting and costovertebral angle tenderness (3). Acute renal colic can be described as a common disorder. The prevalence of kidney stone has roughly tripled in the last 10 years (4).

It is estimated that the risk of stone formation (lithiasis) in an individual is 5 to $10 \%$. The recurrence rate was reported to be $50 \%$ in 5 years and $80-90 \%$ in 10 years after a kidney stone was formed. People who suffer from kidney stone are more likely to have urinary metabolic abnormalities compared to healthy population 
(level of evidence III/C). Patients who have recurrent kidney stones tend to have more significant metabolic abnormalities (5).

Kidney stone (nephrolithiasis) disease is 2-3 times more common in men - It is also more common in adults than in the elderly and prevalence in children is the least. Renal colic pain is often defined as the worst pain experienced by patients (3).

There are several diagnostic methods in the diagnosis of acute flank pain. Among these methods, intravenous (i.v.) urography is no longer preferred because of the low diagnostic value of kidney stone detection, the use of contrast agent, the risk of anaphylaxis and the need for bowel preparation. Another method used in the diagnosis of acute flank pain is ultrasonography. Ultrasonography is not a definitive diagnostic method because it is dependent on the observer and does not recognize some types of kidney stones. In the evaluation of acute flank pain, non-contrast spiral computed tomography (CT) is the method of choice imaging modality with its high sensitivity and specificity. Non-contrast spiral CT has become the method of choice imaging modality in the evaluation of acute flank pain as it identifies ureteral stones with a sensitivity and specificity from $98 \%$ to $100 \%$ regardless of size, location and chemical composition. It also describes extraurinary causes, which accounts for about one third of flank pain, and also, no contrast is used and the procedure can be completed in as short as 5 minutes (2).

Approximately $60 \%$ of the patients with renal colic have severe pain. One of the goals of emergency management related to this disease is to control pain until the obstruction is corrected by spontaneous passage or surgical manipulation (6). Non-steroid anti-inflammatory drugs (NSAIDs) and opioids are often used for analgesia in the treatment of renal colic. Both of them were shown to reduce pain significantly (7).

Acute renal colic should be treated with a NSAID as first-line therapy. If there is insufficient response, an opioid drug should be used for acute second-line treatment. Once the acute pain is alleviated, treatment with an NSAID should be continued (4,810).

Some sources suggest both the use of opioids and NSAIDs alone and also in combination with each other for acute renal colic treatment (11). The most important factors that determine the first choice analgesic are: safety, efficacy and cost, suitability, institutional culture and in addition, patient and clinician preferences $(9,11)$.

Renal colic affects approximately 1.2 million people each year and accounts for $1 \%$ of all emergency room admissions and $1 \%$ of all hospital admissions. On average, emergency physicians treat at least one patient with acute renal colic each day. Often, the emergency physician is the first person to see and evaluate these patients. Emergency physicians should make the correct diagnosis in patients with acute flank pain, give correct treatment to them and guide the patient correctly (12).

Accordingly, our study is planned to guide the physicians to provide the right treatment for the renal colic patients in the emergency department. Pethidine is now abandoned due to its side effect profile. Fentanyl or tramadol has been frequently used as narcotic analgesics in renal colic patients $(10,13)$. The aim of our study is to compare the efficiency of fentanyl and tramadol as analgesics in renal colic patients.

\section{Materials and Methods}

\section{Study Design}

This is a randomized double-blind study, comparing i.v. fentanyl with i.v. tramadol administered to patients with moderate and severe pain due to renal colic. This study was carried out between 03/01/2018 and 10/31/2018 at Atatürk University Research Hospital, the major hospital of the eastern Anatolia region, on patients admitted to the emergency department. Our study is conducted in accordance with "Good Clinical Practice" standards and approved by the ethics committee before it started. Written informed consent was obtained from all patients before enrollment.

\section{Patients}

Patients between the ages of 18-70 were accepted as candidates for the study. The patient selection of our study was based on the work of Sanchez-Carpena et al. (6). According to this study, pain starting from the flank region and spreading to the lateral and genital region, hematuria and kidney stones seen on radiography (X-ray) are determined as the inclusion criteria.

Since renal colic pain is severe and unbearable, study protocols were applied before the laboratory and imaging procedures were performed as in the method applied by Sanchez-Carpena et al. (6). Patients without renal colic were excluded from the study. NSAIDs were applied to all patients as suggested by the literature. Afterwards, patients who needed analgesia were divided into two groups. One group of patients received fentanyl and the other group received tramadol.

The definitive diagnosis of renal colic is determined as the presence of kidney stones in non-contrast spiral abdominal tomography. VAS scores at the time of presentation are recorded for all patients included in the study. Fifty mg of dexketoprofen i.v. (Ufsa Pharmaceutical Industry and Trade Inc.) in $100 \mathrm{cc}$ saline solution was given to patients who agreed to participate in our study, as first-line treatment $(14,15)$. 
Thirty minutes after the first-line treatment, additional analgesia requirement was considered. The rescue medication is planned to be applied earlier when the pain worsens during the first 30 minutes, or if the patient demanded. The VAS score of the patients was asked again 30 minutes after NSAID administration and patients with VAS score 6 and above were given the study drug in 5 minutes in $100 \mathrm{cc}$ isotonic solution via i.v. route. The study drugs, fentanil $1.5 \mathrm{mcg} / \mathrm{kg}$ (Johnson \& Johnson Medical Supplies Industry and Trade Limited Company) (7) and tramadol (Abdi Ibrahim Pharmaceutical Industry and Trade Inc.) were administered for 5 minutes in $100 \mathrm{cc}$ saline solution.

During the study period, 912 patients were admitted to our emergency department with a complaint of renal colic. Five hundred forty-four of these patients did not agree to participate in the study. Six patients were excluded from the study depending on the initial VAS score as $<6$ and 101 patients were excluded from the study due to exclusion criteria. The remaining 261 patients were treated with i.v. NSAID. Hundred of these patients were excluded from the study because of the decrease in pain with NSAIDs (VAS <6) and 18 patients were excluded from the study because they were diagnosed as non-renal colic and our study was completed with 143 patients (Figure 1).

Exclusion criteria were as follows:

- Complicated renal colic,

- Uretero-nephrosis,

- Pyelonephritis,

- General contraindication of NSAID drug use (such as active peptic ulcer),

- Using NSAIDs within 6 hours before application to the emergency department,

- Acute or chronic renal failure,

- Chronic lung disease,

- Heart disease,

- Chronic liver disease,

- Pregnancy or breastfeeding,

- Use of alcohol,

- The presence of bleeding disorder,

- Cognitive impairment or psychiatric illness,

- Cancer patients,

- History of mono amino oxidase inhibitor drugs taken within the last 2 weeks,

- Allergy to opiates,

- Patients who were previously enrolled in the study were not included in the study in their recurrent admissions.

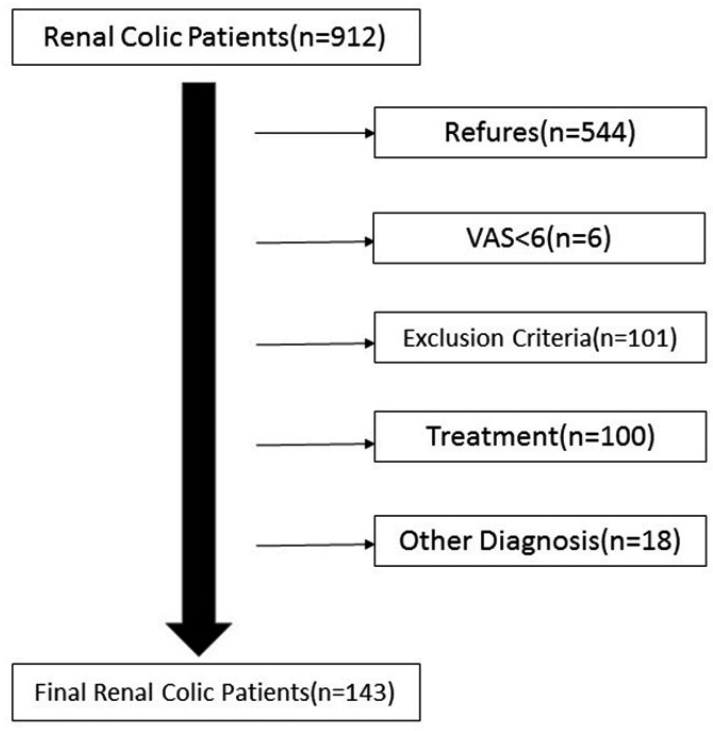

Figure 1. Patient selection scheme for the study

Prior to the study, physicians and nurses were given training about our research. Random separation software was used to determine which patients would be in each group. Gender was not taken into account during randomization because it was not a factor that could affect patients' response to treatment. Patients were included in the study according to the randomization table. Enclosed envelopes, stating which treatment the patient will receive, were prepared by a physician who did not know what treatment to take for each patient number. The drugs to be administered were prepared and packaged by a physician in advance, who did not know the research. The drugs were applied in accordance with the randomization table prepared by the nurse participating in the study. The nurse participating in the study did not know the content of the treatment. The physician who ordered the treatment did not know which drug was applied to the patient too. By this way, the study was provided to be doubleblinded. An emergency medicine specialist was responsible for collecting data who was also blind to the study. Patients were not given any medication other than rescue therapy. The choice of rescue drug is left to the decision of the physician.

\section{Measurement}

Age, sex, vital signs (blood pressure, pulse rate, respiratory rate and fever and oxygen saturation) of the patients who agreed to participate in the study were recorded. Also, data regarding to the time (hour) of pain onset, admission to the hospital (outpatient or by ambulance), and the personal or family history of kidney stone were collected. The VAS score which was assessed 30 minutes after administration of NSAID, was accepted as VAS 0 . The VAS score of the patients at the time of initial admission 
to the hospital is not used in the study. Subsequent times are referred to VAS 0 . Other VAS periods are 5, 15, 30 and 60 minutes after VAS 0.

The primary outcome in the evaluation of analgesic efficiency in our study is the difference between VAS 0 and VAS 60 . Our secondary outcome is that the score given in VAS 60 is 3 or less. Response to treatment is defined as a VAS 60 value below 4 .

\section{Statistical Analysis}

Statistical analyses were performed using SPSS 20 statistical analysis program (IBM). Data are presented as mean, standard deviation, median, minimum, maximum, percentage and number. Normal distribution of continuous variables was assessed using Shapiro-Wilk and Kolmogorov-Smirnov tests. Independent samples t-test was used for comparing normally distributed data between two independent groups and MannWhitney $\mathrm{U}$ test was used to compare non-normally distributed data. Categorical variables were compared using chi-square and Fisher's exact tests. A p value of $<0.05$ was considered statistically significant.

\section{Results}

Our study was completed with the patients admitted to the emergency department and diagnosed with renal colic. Our study was completed with 143 patients. Seventy-one of these patients were grouped as group 1 (fentanyl administered) and 72 of them grouped as group 2 (tramadol administered).

Demographic data of the patients are summarized in Table 1. In our study, there was no significant difference between the groups.

Only 5 of the patients included in our study were admitted to the hospital with an ambulance. In our study, the number of patients who had previously undergone renal colic is 83 in total, with 42 in the fentanyl group and 41 in the tramadol group. In the evaluation of patients with renal colic, 30 patients in the fentanyl group and 32 patients in the tramadol group have family history of renal colic.

The VAS scores $0,15,30,60$ and the difference between VAS scores $0-5,0-15,0-30,0-60$ and the response to the treatment is summarized in Table 2.

Respond to treatment: It is accepted as no response to the treatment if the VAS 60 score was $<4$ after the administration of the study drugs.)

When evaluating the difference between VAS 0 and VAS 60 , which is the primary parameter in the evaluation of the analgesic activity in our study, the mean VAS value in the fentanyl group
Table 1. Demographic data of patients

\begin{tabular}{llll}
\hline & $\begin{array}{l}\text { Group fentanyl } \\
(\mathbf{n}=71)\end{array}$ & $\begin{array}{l}\text { Group tramadol } \\
(\mathbf{n}=72)\end{array}$ & p value \\
\hline Age & $37.87 \pm 13.10$ & $41.24 \pm 12.77$ & $\mathrm{p}>0.05$ \\
\hline Sex (M/F) & $48 / 23$ & $45 / 27$ & $\mathrm{p}>0.05$ \\
\hline $\begin{array}{l}\text { Duration of pain } \\
\text { (mean } \pm \text { standard } \\
\text { deviation) }\end{array}$ & $11.7 \pm 19.6$ & $16.11 \pm 34.35$ & $\mathrm{p}>0.05$ \\
\hline $\begin{array}{l}\text { Duration of pain } \\
\text { (median, minimum, } \\
\text { maximum) }\end{array}$ & $\begin{array}{l}\text { 3, minimum 1, } \\
\text { maximum 72 }\end{array}$ & $\begin{array}{l}\text { 4, minimum 1, } \\
\text { maximum 240 }\end{array}$ & $\mathrm{p}>0.05$ \\
\hline M: Male, F: Female, & & & \\
\hline
\end{tabular}

Table 2. Analysis of patients according to VAS scores (values are presented as number or mean \pm standard deviation

\begin{tabular}{llll}
\hline & $\begin{array}{l}\text { Group } \\
\text { fentanyl }\end{array}$ & $\begin{array}{l}\text { Group } \\
\text { tramadol }\end{array}$ & p value \\
\hline VAS 0 & $8.14 \pm 1.46$ & $8.1 \pm 1.43$ & $\mathrm{p}>0.05$ \\
\hline VAS 5 & $6.27 \pm 2.45$ & $6.1 \pm 2.29$ & $\mathrm{p}>0.05$ \\
\hline VAS 15 & $4.92 \pm 2.69$ & $5.04 \pm 2.44$ & $\mathrm{p}>0.05$ \\
\hline VAS 30 & $3.37 \pm 2.55$ & $3.69 \pm 2.44$ & $\mathrm{p}>0.05$ \\
\hline VAS 60 & $2.03 \pm 2.34$ & $2.15 \pm 2.25$ & $\mathrm{p}>0.05$ \\
\hline VAS difference 0-60 & $6.11 \pm 2.49$ & $5.94 \pm 2.40$ & $\mathrm{p}>0.05$ \\
\hline VAS difference 0-30 & $4.77 \pm 2.56$ & $4.4 \pm 2.43$ & $\mathrm{p}>0.05$ \\
\hline VAS difference 0-15 & $3.23 \pm 2.58$ & $3.06 \pm 2.41$ & $\mathrm{p}>0.05$ \\
\hline VAS difference 0-5 & $1.87 \pm 2.22$ & $2.00 \pm 2.11$ & $\mathrm{p}>0.05$ \\
\hline Response to treatment & $16 / 55$ & $18 / 54$ & $\mathrm{p}>0.05$ \\
\hline
\end{tabular}

was $6.11 \pm 2.49$, while in the tramadol group the VAS value was $5.94 \pm 2.40$. There is no statistically significant difference between the efficiencies of the two drugs.

Our secondary evaluation parameter is that the score given in VAS 60 is 3 or less. This is accepted as response to the treatment. While 16 patients responded to the treatment in the fentanyl group, 18 patients responded to the treatment in the tramadol group. The difference is not statistically significant.

In the analysis made in terms of these two parameters, it is shown that there is no superiority of both drugs on each other. However, our study shows that both drugs are effective in the treatment of renal colic.

During the study, 1 patient in the fentanyl group and 3 patients in the tramadol group required additional analgesic treatment. Side effects were seen in 13 patients in the fentanyl group and in 7 patients in tramadol group. However, this is not statistically significant $(p>0.05)$.

The side effects were; nausea (fentanyl administered group: 11, tramadol administered group: $4 \mathrm{p}>0.05$ ), vomiting (fentanyl administered group: 3, tramadol administered 
group: $3 p>0.05$ ), dizziness (fentanyl administered group: 13, tramadol administered group: $4 p<0.05$ ), hypotension (fentanyl administered group: 2, tramadol administered group: $4 p>0.05$ ). In the analysis of side effect profile, there is significantly more patients experienced dizziness in fentanyl treatment group.

\section{Discussion}

As far as we know, this is the first study evaluating the analgesic efficiency of i.v. fentanyl and i.v. tramadol in renal colic patients. Our results suggest that both of i.v. tramadol and i.v. fentanyl provides effective analgesia in renal colic patients. Although both drugs provide effective analgesia, no superiority is found on each other.

In our study, the VAS 0 score of the fentanyl group is 8.14 while the VAS 0 score of the tramadol group is 8.1 . VAS 60 values are 2.03 and 2.15 respectively. This is an indication of the similar effectiveness of narcotic drugs used in our study.

According to the side effects both drugs were found to have similar properties. The only difference between the two drugs is that fentanyl administration causes significantly more dizziness as a side effect.

Acute flank pain is usually caused by a stone in the ureter (16). Urine excretion decreases due to stone. The urine will then accumulate in the renal pelvis and increase renal pressure. In order to remove the stone, smooth muscles in the urinary system will begin to contract (6). Tension in the kidney pelvis leads to stimulation, synthesis and local release of prostaglandins (3). Prostaglandins increase both the renal blood flow and glomerular filtration rate and consequently the pressure increase in the urinary tract exacerbates pain (6). In addition, these prostaglandins also activate the ureter peristalsis and promote the local inflammatory reaction. Prostaglandin production plays an active role in renal colic (17). If ureteral obstruction is not resolved, kidney failure may occur. The best and most effective treatments for renal colic pain are; spontaneous passage of the stone, removal of the stone, insertion of a stent into the ureter and percutaneous nephrostomy. Fortunately, most patients do not have complete ureteral obstruction and therefore most patients do not face a risk of renal failure (3).

As most kidney stones are spontaneously excreted, the most important point in acute management is the relief of pain and the recognition of complications. Both NSAIDs and opioids reduce pain in acute renal colic. Opioids have the advantages of being cheap, titrable, efficient and familiar, but there are concerns about addiction. Opioids do not directly affect the cause of pain, which can limit their usefulness. NSAIDs act directly on prostaglandin release (the main cause of pain). In particular, they are effective when administered intravenously. However, when compared to opioids, the NSAIDs are generally non-titrable and has well-known side effects such as renal failure and gastrointestinal bleeding (18).

Since the late 1970s, NSAIDs have been widely used alone or in combination with opioids for the treatment of renal colic. Many studies have confirmed the efficacy of NSAIDs, especially when administered i.v. (6). NSAIDs have predominant effects through the inhibition of the cyclooxygenase (COX) enzyme, which regulates the synthesis of autacoids, such as prostaglandins and thromboxane.

As prostaglandin inhibitors, the pharmacological effects of these agents interfere with the underlying renal colic mechanism, rather than suppressing pain. It is concluded that patients who received NSAIDs achieved a further reduction in their pain scores and the likelihood of requiring more analgesia was lower in the short term, when compared to patients treated with opioid (6).

Dexketoprofen trometamol is an anti-inflammatory and analgesic drug that inhibits COX1 and COX2 (1). Dexketoprofen trometamol has a dual pain-relieving effect. Dexketoprofen trometamol has peripheral effect by inhibiting the sensitization of pain receptors induced by locally released prostaglandins. It centrally reduces the effect of central sensitization by inhibiting cox activity. Therefore, it blocks the transfer of the pain stimulus to the upper nerve centers (19). In addition to this direct effect, it also affects other inflammatory mediators such as quinine, creating an indirect effect (20).

Dexketoprofen trometamol has a good analgesic efficacy as well as a good safety profile compared to other NSAIDs in terms of side effects. Furthermore, there is strong evidence that Dexketoprofen trometamol has a faster onset of action $(17,21)$. Dexketoprofen is eliminated very quickly after being metabolized. This situation prevents the accumulation of drugs in healthy adults (20). Dexketoprofen trometamol was selected as the non-steroidal anti-inflammatory drug of choice in our study with all these properties.

Narcotics have been used for pain control in renal colic for a long time. The benefits of opioid use are; low cost, good efficacy, being able to titrate and not causing gastrointestinal bleeding and renal failure. However, the majority of physicians are not comfortable to use these medications because of side effects such as nausea, vomiting, sedation, dizziness, narcotic dependence, disorientation, respiratory depression and hypotension $(3,9)$. Fentanyl is a synthetic $\mu$ receptor stimulating opioid which is a fast-onset opioid with 90 times stronger 
analgesic potential than morphine (22,23). Analgesia may occur 1 to 2 minutes after i.v. fentanyl administration (22). The duration of effect of fentanyl usually lasts from 2 to 4 hours after i.v. or transmucosal administration (24). After administration of fentanyl, constipation and pruritus are less common than morphine. Fentanyl has recently become popular as it has minimal cardiovascular effects, does not cause an increase in plasma histamine, is relatively faster in effect onset and shorter duration of action, easy to synthesize and easy to prepare for market (22). It is also used for analgesia in the treatment of renal colic (13). Tramadol is considered to be the first member of the "atypical opioids" group. Unlike other opioids, tramadol can modulate the reuptake of serotonin (5HT) in the monoaminergic system, noradrenaline and presynaptic terminals. Other effects of tramadol are that $\mathrm{M} 1$ and $\mathrm{M} 3$ muscarinic receptors affect the hyperpolarization of neurons by inhibiting NMDA receptors and opening $\mathrm{K}+$ channels (25).

Although in some academic works $(3,8)$ tramadol has been reported to be ineffective in severe and acute pain; in many other researches, tramadol has been shown to be effective in acute and severe pain $(1,25-27)$. In our study, tramadol was found to be effective in acute and severe pain.

In the studies performed, tramadol was also found to be effective in cases such as neuropathic pain, inflammation, depression, obsessive compulsive disorder, renal colic, cancer pain, joint diseases and low back pain. Tramadol has no effect on cardiovascular and pulmonary parameters. It causes less constipation and opioid-induced intestinal dysfunction and has a low dependency ratio. Tramadol analgesic activity can be further improved in combination with a non-opioid analgesic. Due to preliminary clinical studies in nociception models, first evidence of analgesic synergy between tramadol and dexketoprofen was provided and positive interaction between drugs with a different mechanism of action was demonstrated $(1,25)$.

It is supported by various studies that tramadol and dexketoprofen provide effective analgesia $(28,29)$. In our study, tramadol and dexketoprofen are used as an up-to-date combination. Our study is the first study in which tramadol and dexketoprofen were used together in renal colic patients.

In a study by Engeler et al., (4) urologists in Switzerland were asked what they use for renal colic treatments. In the study, it was concluded that $81 \%$ of urologists used non-opioid analgesics as the first-line treatment. In a double-blind, randomized controlled study, it is found that the combination of i.v. NSAIDs and morphine is superior to the single drug in reducing pain scores (30). However, there is no advantage of opioid use as firstline analgesic (9). In our study, NSAIDs drugs were applied as the first choice in the treatment.
It is reported in many studies that, NSAIDs are more effective than opioids in renal colic patients $(9,11,18)$. In our study, NSAID medication was used as the initial treatment in accordance with the literature. At the end of 30 minutes, the patients who still had pain, were treated with narcotic analgesics (fentanyl, tramadol) as second-line treatment and the results were compared.

In one study, in a group of patients who received i.v. fentanyl for renal colic, the rate of severe pain was $44.4 \%$ after the $5^{\text {th }}$ minute, $28.9 \%$ after the $10^{\text {th }}$ minute and $24.4 \%$ after the $15^{\text {th }}$ minute and $28.9 \%$ after the $30^{\text {th }}$ minute (31). In another study, fentanyl was administered to a renal colic patient and VAS values were determined as VAS 0: 75.56, VAS 15: 36.29, VAS 30: 28.18 (7). In our study, fentanyl effect was found to be higher. In our study, VAS 0: 8.14, VAS 15: 4.92, VAS 30: 3.37.

In the study conducted by Hazhir et al., (32) on the evaluation of the effect of intramuscular tramadol and meperidine, they concluded that the effects of intramuscular $100 \mathrm{mg}$ tramadol and $50 \mathrm{mg}$ meperidine were similar. In our study, tramadol and fentanyl are found to be similar effective for renal colic patients.

\section{Conclusion}

Our study is the first research to compare the efficiency of i.v. fentanyl and i.v. tramadol in renal colic pain. In our study, it is concluded that both drugs are effective on renal colic pain, but they do not have any superiority on each other.

\section{Ethics}

Ethics Committee Approval: Atatürk University, approval number:B.30.2.ATA.0.01.00/70.

Informed Consent: Written informed consent was obtained from all patients before enrollment.

Peer-review: Externally and internally peer-reviewed.

Financial Disclosure: The author declared that this study has received no financial support.

\section{References}

1. Varrassi G, Hanna M, Macheras G, Montero A, Montes Perez A, Meissner W, et al. Multimodal analgesia in moderate-to-severe pain: a role for a new fixed combination of dexketoprofen and tramadol. Curr Med Res Opin. 2017;33(6):1165-73.

2. Heidenreich A, Desgrandschamps F, Terrier F. Modern approach of diagnosis and management of acute flank pain: review of all imaging modalities. Eur Urol. 2002;41(4):351-62.

3. Golzari SE, Soleimanpour H, Rahmani F, Zamani Mehr N, Safari S, Heshmat Y et al. Therapeutic approaches for renal colic in the emergency department: a review article. Anesth Pain Med. 2014;4(1):e16222.

4. Engeler DS, Schmid S, Schmid HP. The ideal analgesic treatment for acute renal colic-theory and practice. Scand J Urol Nephrol. 2008;42(2):137-42. 
5. Skolarikos A, Straub M, Knoll T, Sarica K, Seitz C, Petrik A, et al. Metabolic evaluation and recurrence prevention for urinary stone patients: EAU guidelines. Eur Urol. 2015;67(4):750-63.

6. Sanchez-Carpena J, Dominguez-Hervella F, Garcia I, Gene E, Bugarin R, Martin A, et al. Comparison of intravenous dexketoprofen and dipyrone in acute renal colic. Eur J Clin Pharmacol. 2007;63:751-60.

7. Imamoglu M, Aygun A, Bekar O, Erdem E, Cicek M, Tatli O, et al. A retrospective analysis of nebulized versus intravenous fentanyl for renal colic. Am J Emerg Med. 2017;35:757-63.

8. Micali S, Grande M, Sighinolfi MC, De Carne C, De Stefani S, Bianchi G. Medical therapy of urolithiasis. J Endourol. 2006;20:841-7.

9. Pathan SA, Mitra B, Cameron PA. A Systematic Review and Meta-analysis Comparing the Efficacy of Nonsteroidal Anti-inflammatory Drugs, Opioids, and Paracetamol in the Treatment of Acute Renal Colic. Eur Urol. 2018;73:583-95

10. Guidelines on urolithiasis. European Association of Urology [Internet]. [cited July 1, 2018]. Available from: http://uroweb.org/guideline/urolithiasis/.

11. Holdgate A, Pollock T. Nonsteroidal anti-inflammatory drugs (NSAIDs) versus opioids for acute renal colic. Cochrane Database Syst Rev. 2005:CD004137.

12. Phillips E, Kieley S, Johnson EB, Monga M. Emergency room management of ureteral calculi: current practices. J Endourol. 2009;23:1021-4.

13. Bektas F, Eken C, Karadeniz O, Goksu E, Cubuk M, Cete Y. Intravenous paracetamol or morphine for the treatment of renal colic: a randomized, placebo-controlled trial. Ann Emerg Med. 2009;54:568-74.

14. Cordell WH, Wright SW, Wolfson AB, Timerding BL, Maneatis TJ, Lewis RH, et al. Comparison of intravenous ketorolac, meperidine, and both (balanced analgesia) for renal colic. Ann Emerg Med. 1996;28:151-8.

15. al-Sahlawi KS, Tawfik OM. Comparative study of the efficacy of lysine acetylsalicylate, indomethacin and pethidine in acute renal colic. Eur J Emerg Med. 1996;3:183-6.

16. Davenport K, Waine $E$. The Role of Non-Steroidal Anti-Inflammatory Drugs in Renal Colic. Pharmaceuticals (Basel). 2010;3:1304-10.

17. Sanchez-Carpena J, Sesma-Sanchez J, Sanchez-Juan C, Tomas-Vecina S, GarciaAlonso D, Rico-Salvado J, et al. Comparison of dexketoprofen trometamol and dipyrone in the treatment of renal colic. Clin Drug Investig. 2003;23:139-52.

18. Holdgate A, Pollock T. Systematic review of the relative efficacy of nonsteroidal anti-inflammatory drugs and opioids in the treatment of acute renal colic. BMJ. 2004;328:1401.
19. Mauleon D, Artigas R, Garcia ML, Carganico G. Preclinical and clinical development of dexketoprofen. Drugs. 1996;52 Suppl 5:24-45; discussion -6.

20. Barbanoj MJ, Antonijoan RM, Gich I. Clinical pharmacokinetics of dexketoprofen. Clin Pharmacokinet. 2001;40:245-62.

21. Ceyhan D, Bilir A, Gulec MS. Evaluation of the Analgesic Efficacy of Dexketoprofen Added to Paracetamol. Turk J Anaesthesiol Reanim. 2016;44:312-6.

22. Stanley TH. The fentanyl story. J Pain. 2014;15:1215-26.

23. Ahmadi O, Dehkordi AS, Heydari F, Esfahani MN, Mahaki B. The effect of nitrous oxide in comparison to oxygen combined with fentanyl on the hospitalization time and pain reduction in renal colic patients at emergency department. J Res Med Sci. 2018;23:18.

24. Bailey PL. Intravenous opioid anesthetics. Anesthesia. 1994:291-387.

25. Bravo L, Mico JA, Berrocoso E. Discovery and development of tramadol for the treatment of pain. Expert Opin Drug Discov. 2017;12:1281-91.

26. Affaitati G, Giamberardino MA, Lerza R, Lapenna D, De Laurentis S, Vecchiet L. Effects of tramadol on behavioural indicators of colic pain in a rat model of ureteral calculosis. Fundam Clin Pharmacol. 2002;16:23-30.

27. Lopopolo M, Affaitati G, Fabrizio A, Massimini F, Lapenna D, Giamberardino $\mathrm{MA}$, et al. Effects of tramadol on viscero-visceral hyperalgesia in a rat model of endometriosis plus ureteral calculosis. Fundam Clin Pharmacol. 2014;28:331-41.

28. What place is there for tramadol/dexketoprofen? Drug Ther Bull. 2018;56: 66-8.

29. Derry S, Cooper TE, Phillips T. Single fixed-dose oral dexketoprofen plus tramadol for acute postoperative pain in adults. Cochrane Database Syst Rev. 2016;9:CD012232.

30. Safdar B, Degutis LC, Landry K, Vedere SR, Moscovitz HC, D'Onofrio G. Intravenous morphine plus ketorolac is superior to either drug alone for treatment of acute renal colic. Ann Emerg Med. 2006;48:173-81.

31. Motamed H, Maleki Verki M. Intravenous Lidocaine Compared to Fentanyl in Renal Colic Pain Management; a Randomized Clinical Trial. Emerg (Tehran). 2017;5:e82.

32. Hazhir S, Badr YA, Darabi JN. Comparison of intranasal desmopressin and intramuscular tramadol versus pethidine in patients with renal colic. Urol J. 2010;7:148-51. 